\title{
Measurement of the proton form factor at very low $\mathbf{Q}^{2}$
}

\author{
Adrian Weber*, Miha Mihovilovič and Harald Merkel (for the A1-Collaboration) \\ Institut fuer Kernphysik, Johannes Gutenberg-Universitaet Mainz \\ E-mail: webera@kph.uni-mainz.de
}

\begin{abstract}
After the precise determination of the proton radius via the spectroscopy of muonic hydrogen [2], the proton radius puzzle arose in 2010. The extracted proton radius differs for over 7 standard deviations from the results obtained from electron scattering experiments and hydrogen spectroscopy $[3,4]$. To help understanding this discrepancy an experiment has been performed at the Mainz Microtron (MAMI) in order to measure the proton form factors at the lowest possible values of $\mathrm{Q}^{2}$ to date, using a method based on initial state radiation (ISR). In the following the problem will be briefly discussed and then the ISR approach will be described. Then the analysis will be presented and preliminary results will be shown. At the end an outlook for further exploiting the ISR method will be given.
\end{abstract}

54th International Winter Meeting on Nuclear Physics

25-29 January 2016

Bormio, Italy

\footnotetext{
* Speaker.
} 


\section{Introduction}

For more than 60 years experiments have been performed to determine the charge radius of the proton [5]. The radius has been determined by different electron scattering experiments and also hydrogen spectroscopy [4]. These results produced a consistent value for the proton charge radius. But in 2010 results from the spectroscopy of muonic hydrogen were published and they differed for over 7 standard deviations from the by then accepted value [2], see Fig. 1. This discrepancy which is currently unexplained and known as the proton radius puzzle is a hot topic of today's nuclear physics, because after more than 60 years of research a fundamental property of a basic constituent of matter, its radius, is still not properly known.

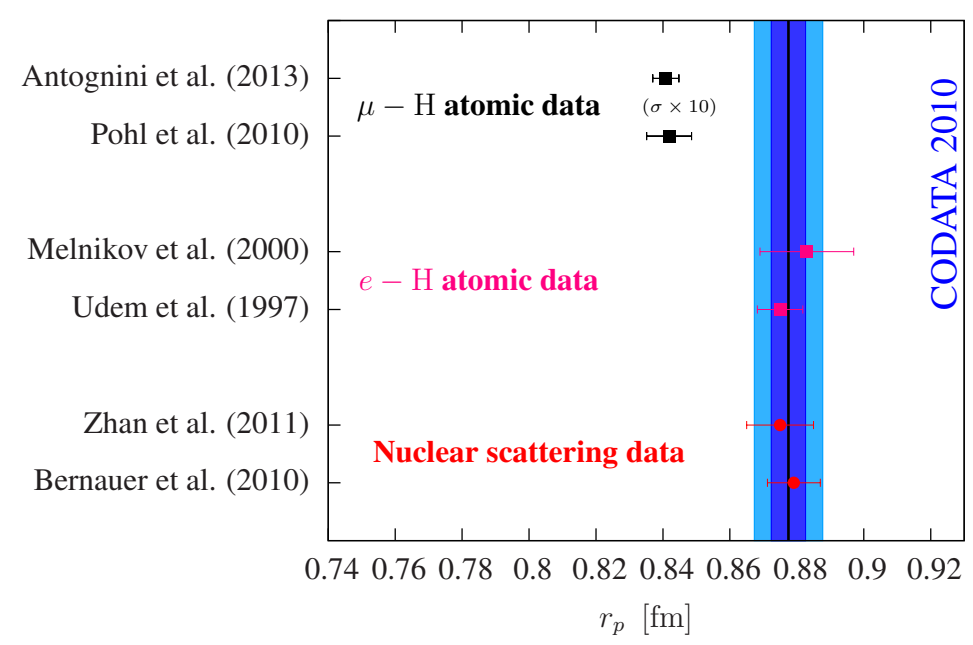

Figure 1: Overview of existing proton charge radius results. Results of the scattering experiments are shown with red circles. Squares represent values obtained spectroscopy measurements. The values determined from the muonic hydrogen measurements are displayed with black squares [6].

Since the occurrence of the puzzle several explanations for the problem were offered $[1,7]$. They ranged from experimental mistakes to missing higher order correction terms in the theoretical calculation to new physics beyond the Standard Model [1]. One theoretical explanation for the discrepancy is that a massless scalar field has disformal interactions with matter particles [10]. The corrections to the Lamb shift thereby depend on the mass of the fermion orbiting the nucleus and imply a larger effect for muonic atoms and would such explain the proton radius puzzle [10]. Another explanation, although it seems unlikely, is the failure of lepton universality. This could be caused by a beyond the Standard Model interaction which couples differently to the muon and the electron [1]. Such an interaction could also explain the muon anomalous magnetic moment [1]. Several experiments are proposed to provide new data for further insides and a possible solution for this puzzle $[1,8,9]$. One of them is a new scattering experiment at MAMI aiming to measure the proton charge form factors at very low $\mathrm{Q}^{2}$, which is the squared momentum transfer four vector, as low as $10^{-4}(\mathrm{GeV} / c)^{2}$, which will be discussed below.

In a typical electron scattering experiment, aiming at determining the proton radius, the elastic cross section is measured at different kinematic settings [11,3]. The cross section depends on the electric and magnetic form factors of the proton, they can be determined from the data using the 
Rosenbluth separation [11]. The charge radius is defined as the slope of the electric form factor at $\mathrm{Q}^{2}=0$ :

$$
\left\langle r_{E}^{2}\right\rangle \equiv-\left.6 \hbar^{2} \frac{d}{d Q^{2}} G_{E}\left(Q^{2}\right)\right|_{Q^{2}=0}
$$

But at the moment no data at very low $\mathrm{Q}^{2}$, which are needed for a precise extraction of the proton charge radius, exist, as can be seen in Fig. 2. And with the available experimental facilities it is not possible to measure at $\mathrm{Q}^{2}<0.004(\mathrm{GeV} / c)^{2}$ by measuring elastic electron proton scattering cross sections. Consequently an extrapolation to $\mathrm{Q}^{2} \rightarrow 0$ has to be done to approximate the slope at $\mathrm{Q}^{2}=0$. But of course the extracted value of the proton charge radius is very sensitive to the model used for the extrapolation. Therefore another method is needed to determine the proton charge form factors at even lower $\mathrm{Q}^{2}$ without building new facilities or change the overall experimental setup.

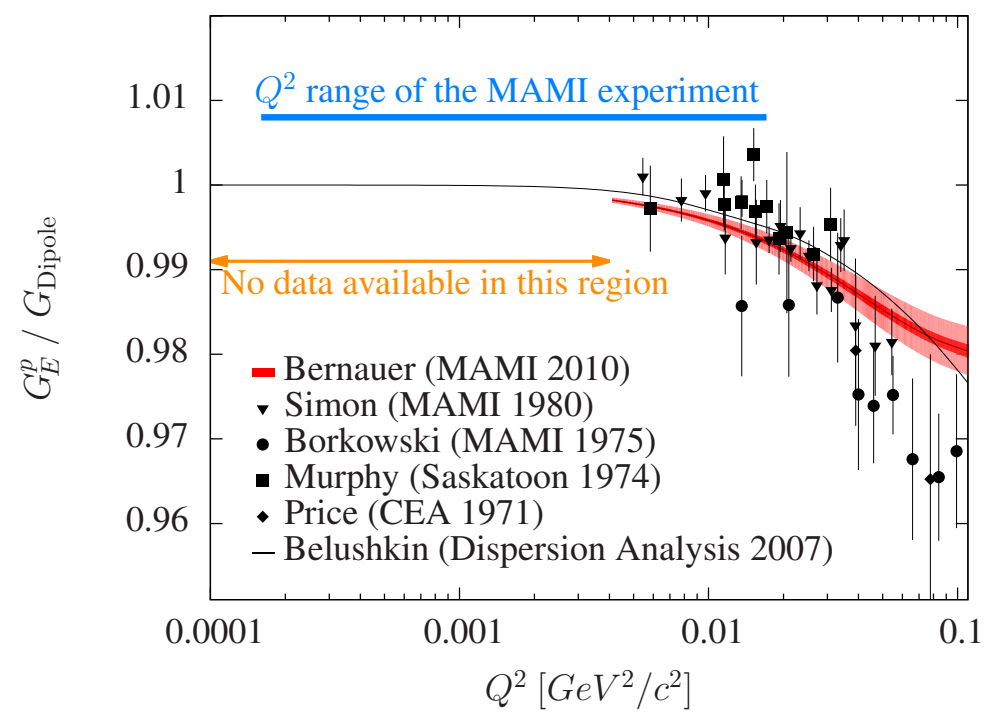

Figure 2: The proton charge form factor $G_{E}^{p}$ normalized to the standard dipole form factor $G_{D}=$ $\left(1-\frac{Q^{2}}{0.71(\mathrm{GeV} / c)^{2}}\right)^{-1}$ as a function of $Q^{2}$. The existing data $[3,12,13,14,15]$ are available only for $Q^{2}>0.004(\mathrm{GeV} / c)^{2}$. The dark red band shows the statistical uncertainty for the fit of the Bernauer data and the light red band shows the uncertainty for the statistical and the systematic uncertainties. The black line shows the results of the dispersion analysis [16]. For a precise determination of the proton charge radius, data at $Q^{2}<0.004(\mathrm{GeV} / c)^{2}$ are needed. The blue band represents the accessible $Q^{2}$ range of the ISR experiment.

\section{Initial State Radiation}

One possible method for reaching lower $\mathrm{Q}^{2}$ is exploiting the radiative tail of the elastic peak. The radiative tail is mostly due to the one photon emission Feynman diagrams for inelastic scattering, see Fig. 3. By measuring the cross section in the radiative tail for a certain kinematic setup it 
is possible to access form factors at lower $\mathrm{Q}^{2}$ values than with the elastic scattering approach. This is mostly due to the ISR process in which the incoming electron emits a photon before the actual scattering process and such reducing the four momentum transfer with respect to elastic scattering under the same conditions. The radiative tail is of course a squared sum of amplitudes of all possible Feynman diagrams. The most important ones are however the initial and final state radiation diagrams. As already explained, the ISR contribution to the radiative tail gives access to lower values of $\mathrm{Q}^{2}$ than the elastic scattering. But the final state radiation has the same $\mathrm{Q}^{2}$ as the elastic scattering and also contributes to the radiative tail. Consequently it is not possible to determine from experimental data alone which events are caused by initial or finial state radiation. Hence a simulation with the underlying Feynman diagrams is needed to be able to determine the amount of events caused by either contribution in a given data set. The simulation also has to calculate the amount of pion production which accounts for up to $10 \%$ of all events. Then the only free parameter which needs to be fitted in the simulation, in order to match data and simulation, is the proton charge form factor. And thus by comparing data and simulation it is possible to extract the proton charge form factors for very low values of $\mathrm{Q}^{2}$.

\section{Experiment}

The actual experiment took place at the MAMI facility in the summer of 2013 at the A1 spectrometer hall. The target was liquid hydrogen which was contained in a havar cell. The MAMI accelerator delivered a continuous beam of electrons. Data were taken for three beam energies: $495 \mathrm{MeV}, 330 \mathrm{MeV}$ and $195 \mathrm{MeV}$. The lowest beam energy is the one which allows to determine the charge form factor down to $\mathrm{Q}^{2} \approx 10^{-4}(\mathrm{GeV} / c)^{2}$, this value is limitted by the minimum beam energy, the minimum angle of the spectrometer and the minimum magnetic field of the spectrometer. The two higher beam energies are studied to validate the ISR method in a region where the form factors have already been measured. Two high resolution spectrometers, which both have an momentum resolution of $\frac{\Delta p}{p}=10^{-4}$, were used to detect scattered electrons [17]. The spectrometer which was set to the smallest possible scattering angle of $15.25^{\circ}$ was used for the actual measurement of electrons scattering elastically and inelastically off protons producing the elastic peak and the radiative tail. This spectrometer has a momentum accetance of $15 \%$. To measure the radiative tail, several setups were recorded. Each setup had an overlap of the momentum acceptance of one half with its neighbouring setup. The other spectrometer was utilised as a luminosity monitor and measured electrons scattering elastically off proton, it has a momentum acceptance of $20 \%$. The conditions of both spectrometers, apart from the magnetic field change of the spectrometer measuring the radiative tail, were kept constant during the entire experiment in order to minimize sources for systematic uncertainty.

\section{Simulation}

The simulation required for calculating the Feynman diagrams shown in Fig. 3 follows Ref. [18], and is included in the simulation environment of the A1 experimental setup at MAMI. This simulation exactly calculates the amplitudes for the four Feynman diagrams presented in Fig. 3, while 


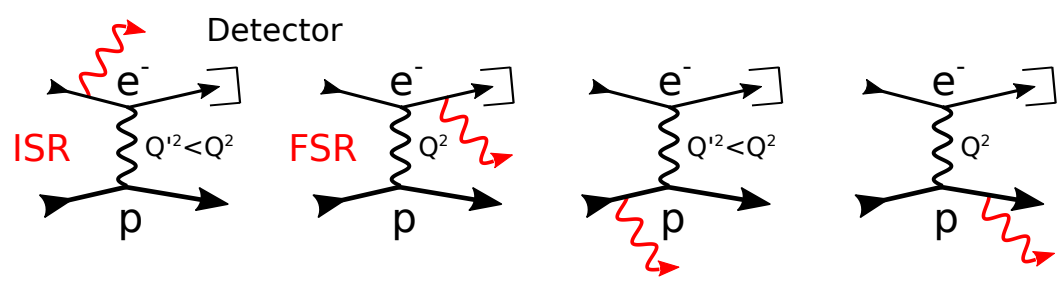

Figure 3: First order Feynman diagrams for inelastic scattering. In the Bethe-Heitler processes a real photon is emitted by the electron: ISR is the initial state radiation and FSR is the final state radiation. In the Born processes a real photon is emitted by the proton. During the experiment the scattered electron is detected.

higher order diagrams are implemented as effective corrections which are accurate to $1 \%$ and contribute up to $30 \%[18,19,20,21]$. The simulation also includes the specifics of the A1 experimental setup, like the acceptances of the spectrometers, and can such be used to compare data and simulation. In Fig. 4 the results of the simulation for a setup with $195 \mathrm{MeV}$ beam energy is displayed. On the abscissa the energy of the scattered electrons is plotted. This observable is actually measured during the experiment. On the ordinate $\mathrm{Q}^{2}$ is plotted. This variable is only accessible in the simulation. By combining the simulation with the measured data it is possible to correctly disentangle the initial and final state radiation contributions. The simulation is also capable of simulating contributions arising from electrons scattered off the target cell walls containing the liquid hydrogen, which is detected as background to the desired data. And in addition it is possible to simulate detected electrons which are due to the production of neutral or positive pions [22], too. The proton form factor distribution is then fitted in the combined simulation to match the data.

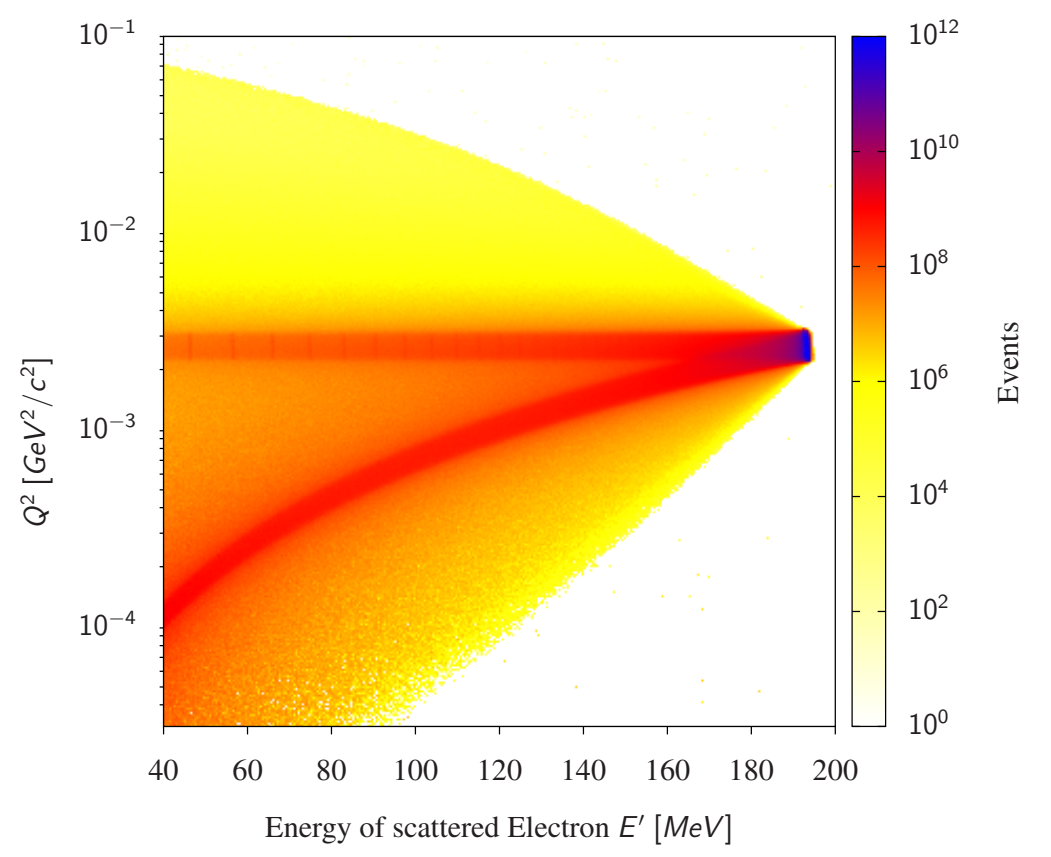

Figure 4: Results of the simulation for an experimental setup with a beam energy of $195 \mathrm{MeV}$. The plot shows the detected rates as a function of $Q^{2}$ at the vertex, this quantity is only available in the simulation, and the energy of the scattered electron, which is directly measured by the spectrometer. The diagonal and horizontal red curves are caused by initial state and final state radiation respectively. 


\section{Analysis}

During the first part of the offline analysis the efficiencies of the detectors and important observables concerning the analysis like the density of the liquid hydrogen and the stability of the magnetic field were examined. The efficiencies of the detectors were all in the order of greater than 99\%. As an example the efficiency for the tracking detectors, which are vertical drift chambers, is presented in Fig. 5. There the overall efficiency is close to $100 \%$. Also the density of the liquid hydrogen was determined to be almost constant during the entire experiment and the magnetic field had a relative stability better than $\frac{\Delta p}{p}=10^{-4}$. In the following part of the analysis the simulation for the background contribution from the target cell was optimized. Some cuts were introduced to reduce the background from the target cell walls as well. Then the combined simulation for the electron-proton scattering cross section, the background simulation and the pion simulation was run together. Preliminary results are shown in Fig. 6. It has to be noted that in the simulation the form factors obtained in Ref. [3] were used. In the lower part of the plot the difference between the simulation and the data is displayed. Using the form factors given in Ref. [3], data and simulation already match within about $1 \%$. This result already validates the application of the ISR method, as in the overlapping parts of the different energy setups the results agree within the error bars. The error bars of the data points are the statistical errors. The plotted systematic uncertainties are combined from different sources. The events from the background simulation have an uncertainty of 5\%, also the events from the pion simulation have an uncertainty of 5\%, the electron proton scattering cross section calculation has an error of $0.36 \%$ due to due higher order corrections, the efficiency of the detectors has been determined with an uncertainty of $0.2 \%$ and the determination of the luminosity has an uncertainty of $0.17 \%$. Due to a huge amount of background contribution in for a large fraction of the data at $195 \mathrm{MeV}$, this sample could not be included in the analysis, because the background contribution could not be estimated precisely enough. Therefore with this experiment it is only possible to determine the proton charge form factors for values of

$\mathrm{Q}^{2} \geq 1.3 \cdot 10^{-3}(\mathrm{GeV} / \mathrm{c})^{2}$. The determination of the actual form factor values and the extraction of the proton charge radius are ongoing. 


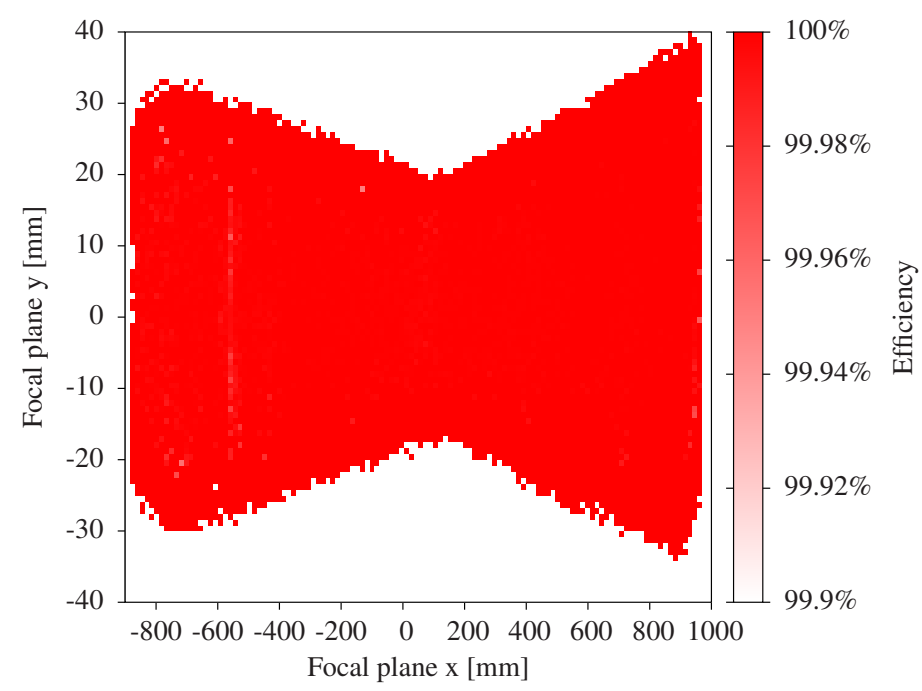

Figure 5: Efficiency of the vertical drift chambers as a function of the coordinates at the focal plane.

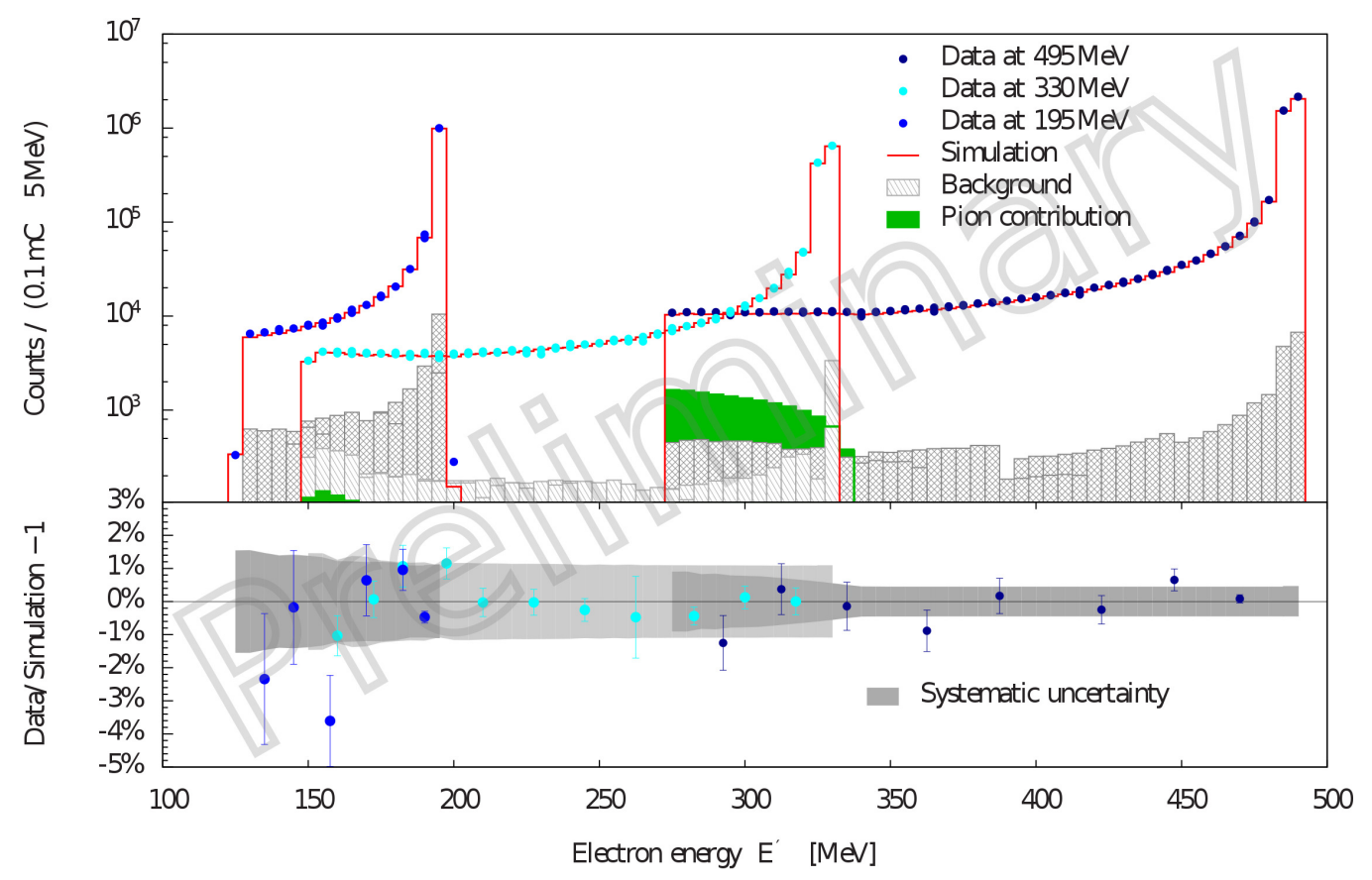

Figure 6: Preliminary results of the ISR analysis. Data and simulation match in the overlapping region of two energy setups and therefore validate the ISR method.

\section{Conclusion and outlook}

The proton radius puzzle is still an open question in nuclear physics. By using a method based on ISR it is possible to determine the proton charge form factors at very low values of $\mathrm{Q}^{2}$. These are needed in order to determine the proton charge radius with very high precision and independent of the underlying fitting model. During the described experiment background contributions appeared, preventing the determination of the proton charge form factors at the lowest accessible 
$\mathrm{Q}^{2}$ of $10^{-4}(\mathrm{GeV} / c)^{2}$ with the required precision. Hence, to exploit the complete potential of the ISR method at MAMI a new experiment is already being commissioned. For this future experiment a gas jet target, see Fig. 7, will be used instead of a liquid hydrogen target. This will completely elimate the background contribution arising from the target cell and allow to determine the proton charge form factors down to $\mathrm{Q}^{2} \approx 10^{-4}(\mathrm{GeV} / c)^{2}$ with high precision.

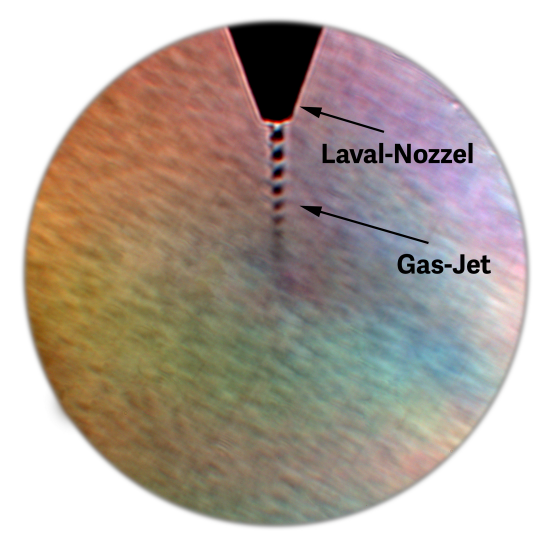

Figure 7: Picture of the density profile of the gas jet target, obtained with a diffraction photographic technique.

\section{Acknowledgments}

This work is supported by the Federal State of Rhineland-Palatinate and by the Deutsche Forschungsgemeinschaft with the Collaborative Research Center 1044.

\section{References}

[1] R. Pohl et al., Annu. Rev. Nucl. Part. Sci. 63, 175 (2013).

[2] R. Pohl et al., Nature 466, 213 (2010).

[3] J. C. Bernauer et al., Phys. Rev. Lett. 105, 242001 (2010).

[4] J. C. Bernauer and R. Pohl. Scientific American 310, 32 (2014).

[5] R. W. McAllister and R. Hofstadter, Phys. Rev. 102 , 851 (1956).

[6] J. Beringer et al. (Particle Data Group), Phys. Rev. D 86, 010001 (2012).

[7] I. Sick, Prog. Part. Nucl. Phys. 67, 473 (2012).

[8] M. Meziane et al., AIP Conf. Proc. 1563, 183 (2013).

[9] R. Gilman et al., arXiv:1303.2160 [nucl-ex] (2013).

[10] P. Barx et al., Phys. Rev. D 91, 043515 (2015)

[11] M. N. Rosenbluth, Phys. Rev. 79, 615 (1950).

[12] G. G. Simon et al., Nucl. Phys. A 333, 381 (1980).

[13] F. Borkowski et al.., Nucl. Phys. B 93, 461 (1975). 
[14] J. J. Murphy et al., Phys. Rev. C 9, 2125 (1974).

[15] L. E. Price et al., Phys. Rev. D 4, 45 (1971).

[16] M. A. Belushkin et al., Phys. Rev. C 75, 035202 (2007).

[17] K. I. Blomqvist et al., Nucl. Instr. Meth. Phys. Res. A 403, 263 (1998)

[18] M. Vanderhaeghen et al., Phys. Rev. C 62, 025501 (2000).

[19] L. C. Maximon and J. A. Tjon, Phys. Rev. C 62, 054320 (2000).

[20] Y. S. Tsai, Phys. Rev. 122, 1898 (1960).

[21] L. W. Mo and Y. S. Tsai, Rev. Mod. Phys. 41, 205 (1969).

[22] D. Drechsel et al., Eur. Phys. J. A. 34, 69 (2007). 